doi:10.13108/2021-13-2-15

\title{
ON INTEGRABILITY OF SEMI-DISCRETE TZITZEICA EQUATION
}

\author{
R.N. GARIFULLIN
}

Abstract. In the paper we consider a semi-discrete version of Tzitzeica equation

$$
\frac{d u_{n+1}}{d x}=\frac{d u_{n}}{d x}+\left(e^{-2 u_{n}}+e^{-2 u_{n+1}}\right)+\sqrt{e^{2 u_{n}}+e^{2 u_{n+1}}},
$$

which was found in a recent paper [R.N. Garifullin and I.T. Habibullin 2021 J. Phys. A: Math. Theor. 54 205201]. It was shown that this equation possessed generalized symmetries along the discrete and continuous directions. These generalized symmetries are equations of Sawada-Kotera equation type and of discere Sawada-Kotera equation type. In this work we construct the Lax pair for this equation and for its generalized symmetries. The found Lax pair is written out in terms of $3 \times 3$ matrices and this indicates the integrability of the found equations. To solve this problem, we employ the known relation between one of the generalized symmetries with a well-studied Kaup-Kupershmidt equation. The found Lax pairs can be employed in further studies of this equation, namely, for finding its conservations laws, the recursion operators and wide classes of solutions. Moreover, we write out two Lax representations in the form of scalar operators. The first representation is written in terms of the powers of the differentiation operators with respect to the continuous variable $x$, while the other is written via the powers of the operator of the shift along the discrete variable $n$.

Keywords: integrability, Lax pairs, generalized symmetries, Tzitzeica equation.

Mathematics Subject Classification: 39A14, 39A10, 35L10

\section{INTRODUCTION}

In a recent paper [1], there was found a differential-difference equation

$$
u_{n+1, x}=u_{n, x}+\lambda_{1}\left(e^{-2 u_{n}}+e^{-2 u_{n+1}}\right)+\lambda_{2} \sqrt{e^{2 u_{n}}+e^{2 u_{n+1}}} .
$$

Here an unknown function $u_{n}(x)$ depends on one integer variable $n \in \mathbb{Z}$ and one continuous variable $x, \lambda_{1}, \lambda_{2}$ are non-zero parameters and without loss of generality we can assume they both are equal to 1 . Hereinafter by $u_{n, x}$ we denote the derivatives in the variable $x$. In the same way we denote the derivatives in $t$ and $\tau: u_{n, t}, u_{n, \tau}$.

In a continuous limit, equation (1.1) obviously transforms into a famous Tzitzeica equation [2]

$$
U_{x y}=a e^{-2 U}+b e^{U},
$$

which was re-discovered in work by A.V. Zhiber and A.B. Shabat [3]. Equation (1.1) is a representative of the class of equations of form

$$
u_{n+1, x}=f\left(u_{n, x}, u_{n+1}, u_{n}, x\right) .
$$

R.N. Garifullin, On integrability of Semi-Discrete Tzizeica equation.

(C) Garifullin R.N. 2021.

The research is supported by Russian Science Foundation project no. 21-11-00006.

Submitted April 25, 2021. 
Integrable equations of such kind arise as Bäcklund transform for nonlinear evolutionary partial differential equations. The most famous representative of this class is a dressing chain, a detailed study of which was made in paper by A.P. Veselov and A.B. Shabat [4]

$$
u_{n+1, x}=u_{n, x}+u_{n+1}^{2}-u_{n}^{2},
$$

which arose as the Bäcklund transform for a modified Korteweg-de Vries equation:

$$
u_{n, t}=u_{n, x x x}-6 u_{n}^{2} u_{n, x} .
$$

On the other hand, equation (1.4) can be treated as a generalized symmetry of equation (1.3). In the discrete direction, a generalized symmetry of equation 1.3 is of the form

$$
u_{n, \tau}=\frac{\left(u_{n+1}-u_{n}\right)\left(u_{n}-u_{n-1}\right)}{u_{n+1}-u_{n-1}}
$$

and is a known differential-difference equation [5], [6]. In paper [7], R.I. Yamilov provided a series of triples of equations of form (1.3) 1.5 .

Equation (1.1) considered in the present paper is a first example, the generalized symmetries of which in both directions are of fifth order and not of the third order:

$$
\begin{aligned}
\partial_{t} u_{0}= & u_{0,5}+5 u_{0,3}\left(u_{0,2}-u_{0,1}^{2}-\lambda_{2}^{2} e^{2 u}-\lambda_{1}^{2} e^{-4 u}\right)-5 u_{0,2}^{2} u_{0,1} \\
& -15 u_{0,2} u_{0,1}\left(\lambda_{2}^{2} e^{2 u}-4 \lambda_{1}^{2} e^{-4 u}\right)+u_{0,1}^{5}-90 \lambda_{1}^{2} u_{0,1}^{3} e^{-4 u}+5 u_{0,1}\left(\lambda_{2}^{2} e^{2 u}+\lambda_{1}^{2} e^{-4 u}\right)^{2}, \\
\partial_{\tau} u= & \left(\left(v^{2}-1\right)^{2}-4 v_{-1}^{2} T^{-1}\right) \frac{\left(v_{1}^{2}+1\right)\left(v_{-1}^{2}+1\right)}{\left(v^{2}\left(v_{-1}+1\right)^{2}+\left(v_{-1}-1\right)^{2}\right)\left(v_{1}(v+1)^{2}+(v-1)^{2}\right)}, \\
v_{n}= & \sqrt{1+e^{2\left(u_{n}-u_{n+1}\right)}}+e^{u_{n}-u_{n+1}},
\end{aligned}
$$

where $T$ is the shift operator along the discrete variable $n$. Hereinafter we employ the notations $u=u_{n}, u_{k}=u_{n+k}, v_{k}=v_{n+k}, u_{k, m}=\frac{d^{m} u_{n+k}}{d x^{m}}$. As the order of an evolutionary equation we mean the number of derivatives with respect to the spatial variable or the number of the shifts $u_{n+i}$ in the right hand side.

Equation (1.6) was known before [8], while equations (1.1) and (1.7) were first provided in paper [1]. In the present work we find Lax pairs for all these three equations. The Lax pairs for integrable equations are one of the most important attributes. First, their presence is the most recognized criterion for integrability. Second, Lax pairs help in the study of equations: finding their conservation laws, generalized symmetries, recursion operators, and wide classes of solutions.

\section{FINDING OF LAX PAIRS}

Finding at once the Lax pair for equation (1.1) is a difficult problem. In order to solve it, we have to find simultaneously two unknown linear operators or two matrices, the structure of dependence on variables of which is not known a priori. This is why, first we find a Lax pair for the well-known equation (1.6), for which, moreover, Miura-type transformations to a well-studied equation are known.

2.1. Lax pair for equation (1.6). In order to solve this problem, we employ well-known relation [8]

$$
w=-u_{0,2}-u_{0,1}^{2}-\lambda_{1}^{2} e^{-4 u}-\lambda_{2}^{2} e^{2 u}
$$

between equation (1.6) and Kaup-Kuperschmidt equation [9]

$$
w_{t}=w_{5}+10 w w_{3}+25 w_{x} w_{2}+20 w^{2} w_{x} .
$$

Equation (2.2) has Lax representation [9], 10]

$$
\widetilde{L}_{t}=[\widetilde{L}, \widetilde{A}],
$$


where

$$
\begin{aligned}
& \widetilde{L}=\partial^{3}+2 w \partial+w_{x}, \\
& \widetilde{A}=9\left(\widetilde{L}^{5 / 3}\right)_{+}=9 \partial^{5}+30 w \partial^{3}+45 w_{x} \partial^{2}+5\left(4 w^{2}+7 w_{2}\right) \partial+10\left(w_{3}+2 w w_{1}\right) .
\end{aligned}
$$

Here $\partial$ denotes the operator of differentiation in $x$, the notation ()$_{+}$stands for the positive part of a formal series in powers of the operator $\partial$.

If we substitute change (2.1) into Lax representation (2.4), then the obtained operator are compatible on solutiuons of (1.6), but compatibility condition (2.3) does not imply equations (1.6), but instead, it gives some differential implication of this equation. In order to obtain a true Lax representation for equation (1.6), we observe that the operator $\widetilde{L}$ in the variable $u$ admits the following factorization:

$$
\widetilde{L}=\left(\partial-u_{0,1}+\lambda_{1} e^{-2 u}\right)\left(\partial^{2}+\left(u_{0,1}-\lambda_{1} e^{-2 u}\right) \partial+u_{0,2}+2 \lambda_{1} u_{0,1} e^{-2 u}-\lambda_{2}^{2} e^{2 u}\right)+\lambda_{1} \lambda_{2}^{2} .
$$

Then as the operator $L$ for equation (1.6), we can use the operator

$$
L=\left(\partial^{2}+\left(u_{0,1}-\lambda_{1} e^{-2 u}\right) \partial+u_{0,2}+2 \lambda_{1} u_{0,1} e^{-2 u}-\lambda_{2}^{2} e^{2 u}\right)\left(\partial-u_{0,1}+\lambda_{1} e^{-2 u}\right)+\lambda_{1} \lambda_{2}^{2} .
$$

The operator $A$ becomes:

$$
\begin{aligned}
A= & 9\left(L^{5 / 3}\right)_{+}=9 \partial^{5}-15\left(u_{0,2}+u_{0,1}^{2}+\lambda_{1}^{2} e^{-4 u}+\lambda_{2}^{2} e^{2 u}\right) \partial^{3}-15\left(2 u_{0,3}+4 u_{0,2} u_{0,1}\right. \\
& \left.-8 \lambda_{1}^{2} u_{0,1} e^{-4 u}+\lambda_{2}^{2} e^{2 u} u_{0,1}\right) \partial^{2}-5\left(5 u_{0,4}+10 u_{0,3} u_{0,1}-2 u_{0,2} u_{0,1}^{2}+9 u_{0,2}^{2}-u_{0,1}^{4}\right. \\
& \left.-\left(\lambda_{1}^{2} e^{-4 u}+\lambda_{2}^{2} e^{2 u}\right)^{2}+2 \lambda^{2}\left(39 u_{0,2}-11 u_{0,1}^{2}\right) e^{-4 u}-u_{0,2} \lambda_{2}^{2} e^{2 u}\right) \partial-10\left(u_{0,5}+2 u_{0,1} u_{0,4}\right. \\
& +5 u_{0,2} u_{0,3}-u_{0,1}^{2} u_{0,3}-2 u_{0,1} u_{0,2}^{2}-2 u_{0,1}^{3} u_{0,2}-\lambda_{2}^{2} e^{2 u}\left(2 u_{0,3}+7 u_{0,2} u_{0,1}+3 u_{0,1}^{3}\right) \\
& \left.-5 \lambda_{1}^{2} e^{-4 u}\left(u_{0,3}-10 u_{0,2} u_{0,1}+12 u_{0,1}^{3}\right)+u_{0,1}\left(4 \lambda_{1}^{4} e^{-8 u}+5 \lambda_{1}^{2} \lambda_{2}^{2} e^{-2 u}+\lambda_{2}^{4} e^{4 u}\right)\right) .
\end{aligned}
$$

The following statement holds.

Theorem 2.1. Equation (1.6) is equivalent to Lax representation

$$
L_{t}=[L, A],
$$

where the operators $L$ and $A$ are defined by expressions (2.5) and (2.6).

Proof. By straightforward calculations we confirm that equation (2.7) holds on solution of (1.6).

In order to confirm the inverse statement, we note that the coefficients

$$
l_{1}=-u_{0,2}-u_{0,1}^{2}-\lambda_{1}^{2} e^{-4 u}+\lambda_{2}^{2} e^{2 u}
$$

and $l_{0}$ of the operator $L$ at corresponding powers of the operator $\partial$ are related by the identity

$$
l_{0}=\partial l_{1}+3 u_{0,1} \lambda_{2}^{2} e^{2 u} .
$$

This is why 2.7 we find the expressions for $\partial_{t}\left(l_{1}\right)$ and $\partial_{t}\left(u_{0,1} e^{2 u}\right)$. Combining these expressions, we can find uniquely $u_{t}$ and obtain equation (1.6). The proof is complete.

2.2. Lax pair for equation (1.1). Equation (1.1) should have Lax representation

$$
(T L) M-M L=0,
$$

with some operator $M$, where $T$ is the shift operator along the discrete variable $n$. However, apriori we know neither the way of finding the operator $M$, no its order and the structure of the dependence of its coefficients. This is why instead of finding this operator we first pass to a matrix form of the operators $L$ and $A$. It is clear how find a matrix form of the operator $M$ for these operators. In order to do this, we introduce a scalar function $P$ and a spectral parameter $\lambda$ and we write two linear problems

$$
L P=\lambda P, \quad P_{t}+A P=0,
$$


and their compatibility condition is equivalent to equation 1.6. These two problems can be rewritten in the matrix form

$$
\frac{d V}{d x}=\mathcal{L} V, \quad \frac{d V}{d t}=\mathcal{A} V .
$$

Here $V$ is some vector function, while $\mathcal{L}$ and $\mathcal{A}$ are some matrices. The operator $L$ is of third order and this is why the size of the matrices should be $3 \times 3$. Choosing a vector function $V$, we can find the matrices $\mathcal{L}$ and $\mathcal{A}$ corresponding to scalar operators $L$ and $A$. The matrix $\mathcal{L}$ corresponding to the operator $L$ is of the simplest form

$$
\mathcal{L}=\left(\begin{array}{ccc}
-\lambda_{1} e^{-2 u} & 0 & -e^{u} \\
0 & \lambda_{1} e^{-2 u} & e^{u} \\
\frac{\lambda-\lambda_{1} \lambda_{2}^{2}}{2 \lambda_{1}} e^{u} & \frac{\lambda+\lambda_{1} \lambda_{2}^{2}}{2 \lambda_{1}} e^{u} & 0
\end{array}\right)
$$

for the vector function

$$
V=\left(\begin{array}{c}
\lambda_{1} P-e^{2 u} P_{x} \\
\lambda_{1} P+e^{2 u} P_{x} \\
e^{u} P_{x x}+2 u_{0,1} e^{u} P_{x}-\lambda_{1}^{2} e^{-3 u} P
\end{array}\right) .
$$

The matrix $\mathcal{A}$ corresponding to the operator $A$ reads as

$$
\begin{aligned}
\mathcal{A}= & \left(\begin{array}{ccc}
\mathcal{A}_{11} & \frac{9 \lambda\left(\lambda+\lambda_{1} \lambda_{2}^{2}\right)}{2 \lambda_{1}} & \mathcal{A}_{13} \\
-\frac{9 \lambda\left(\lambda-\lambda_{1} \lambda_{2}^{2}\right)}{2 \lambda_{1}} & -\mathcal{A}_{11}-\mathcal{A}_{33} & \mathcal{A}_{23} \\
\mathcal{A}_{31} & \mathcal{A}_{32} & \mathcal{A}_{33}
\end{array}\right), \\
\mathcal{A}_{11}= & \frac{\left(3 \lambda+2 \lambda_{1} \lambda_{2}^{2}\right)\left(3 \lambda-\lambda_{1} \lambda_{2}^{2}\right)}{2 \lambda_{1}} e^{2 u}-3 \lambda\left(u_{0,2}+u_{0,1}^{2}\right)-\lambda_{1}^{2}\left(3 \lambda+2 \lambda_{1} \lambda_{2}^{2}\right) e^{-4 u} \\
& +\lambda_{1}\left(2 u_{0,4}+4 u_{0,3} u_{0,1}+3 u_{0,2}^{2}-2 u_{0,2} u_{0,1}^{2}-u_{0,1}^{4}\right) e^{-2 u}-10 \lambda_{1}^{3}\left(u_{0,2}-3 u_{0,1}^{2}\right) e^{-6 u} \\
& -\lambda_{1} \lambda_{2}^{2}\left(7 u_{0,2}+12 u_{0,1}^{2}\right)-\lambda_{1}^{5} e^{-10 u}, \\
\mathcal{A}_{13}= & -\left(\left(9 \lambda+12 \lambda_{1} \lambda_{2}^{2}\right) u_{0,1}+u_{0,4}-u_{0,3} u_{0,1}+3 u_{0,2}^{2}-4 u_{0,2} u_{0,1}^{2}+u_{0,1}^{4}\right) e^{u} \\
& -\lambda_{1}\left(9 \lambda-3 u_{0,3}-6 u_{0,2} u_{0,1}+2 \lambda_{1} \lambda_{2}^{2}\right) e^{-u}+2 \lambda_{1}^{2}\left(u_{0,2}-15 u_{0,1}^{2}\right) e^{-3 u} \\
& -12 \lambda_{1}^{3} u_{0,1} e^{-5 u}-\lambda_{1}^{4} e^{-7 u}+5 \lambda_{2}^{2} u_{0,2} e^{3 u}-\lambda_{2}^{2} e^{5 u}, \\
\mathcal{A}_{23}= & -\mathcal{A}_{13}+6 \lambda_{1}\left(\left(u_{0,3}+2 u_{0,2} u_{0,1}-3 \lambda\right) e^{-u}-4 u_{0,1}\left(\lambda_{1}^{2} e^{-5 u}+\lambda_{2}^{2} e^{u}\right)\right), \\
\mathcal{A}_{31}= & \frac{\lambda-\lambda_{1} \lambda_{2}^{2}}{2 \lambda_{1}}\left(\mathcal{A}_{23}+18 \lambda\left(\lambda_{1} e^{-u}-u_{0,1} e^{u}\right)\right), \\
\mathcal{A}_{32}= & -\frac{\lambda+\lambda_{1} \lambda_{2}^{2}}{2 \lambda_{1}}\left(\mathcal{A}_{13}+18 \lambda\left(\lambda_{1} e^{-u}+u_{0,1} e^{u}\right)\right), \\
\mathcal{A}_{33}= & 3 \lambda\left(2 u_{0,2}+2 u_{0,1}^{2}+2 \lambda_{1}^{2} e^{-4 u}-\lambda_{2}^{2} e^{2 u}\right) .
\end{aligned}
$$

The compatibility condition of matrix linear problems 2.10

$$
\mathcal{L}_{t}-\mathcal{A}_{x}+[L, A]=0
$$

is equivalent to equation (1.6).

We proceed to constructing of the Lax pair for equation (1.1). To do this, we have one linear problem in the variable $x$, see 2.10$)$, and it remains to find the matrix $\mathcal{M}$ defining the shift of the vector function $V$ :

$$
V_{n+1}=\mathcal{M} V_{n}
$$

Since the matrix $\mathcal{L}$ depends only on $u_{n, 0}$, the matrix $\mathcal{M}$ depends on the variables $u_{n, 0}, u_{n+1,0}$. The compatibility condition is as follows:

$$
\mathcal{M}_{x}+\mathcal{M L}-(T \mathcal{L}) \mathcal{M}=0 .
$$


It should be satisfied due to equation (1.1). The matrix $\mathcal{M}$ is found by this condition and is of the form:

$$
\mathcal{M}=\left(\begin{array}{ccc}
\left(\lambda-\lambda_{1} \lambda_{2}^{2}\right)\left(e^{u_{1}-u}+e^{u-u_{1}}\right) & \left(\lambda+\lambda_{1} \lambda_{2}^{2}\right) e^{u-u_{1}} & 2 e^{u-u_{1}} \lambda_{1} \lambda_{2} \sqrt{e^{2 u_{1}-2 u}+1} \\
-\left(\lambda-\lambda_{1} \lambda_{2}^{2}\right) e^{u_{1}-u} & 0 & 0 \\
-\left(\lambda-\lambda_{1} \lambda_{2}^{2}\right) \lambda_{2} \sqrt{e^{2 u_{1}-2 u}+1} & 0 & \lambda-\lambda_{1} \lambda_{2}^{2}
\end{array}\right) .
$$

It is straightforward to confirm that compatibility condition 2.14 with given matrices $\mathcal{L}, \mathcal{M}$ is equivalent to semi-discrete Tzitzeica equation (1.1):

Theorem 2.2. The Lax pair for semi-discrete Tzitzeica equation is of form (2.14) with matrices (2.11) and 2.15).

Once we have found the matrix $\mathcal{M}$, we can also write the operator $M$ for solvability condition (2.8):

$$
M=\left(\partial+\lambda_{2} \sqrt{e^{2 u_{1}}+e^{2 u}}\right)\left(\partial^{2}-u_{0,2}-u_{0,1}^{2}-\lambda_{1}^{2} e^{-4 u}\right) .
$$

2.3. Lax pair for equation 1 .7). It remains to find the matrix $\mathcal{B}$ determining linear problem in $\tau$ :

$$
\frac{d V}{d \tau}=B V
$$

In this case the compatibility condition

$$
\mathcal{M}_{\tau}+\mathcal{M B}-T(\mathcal{B}) \mathcal{M}=0
$$

should be satisfied due to equation (1.7). The matrix $\mathcal{B}$ is found by this condition:

$$
\begin{aligned}
& \mathcal{B}=\left(\begin{array}{ccc}
-\mathcal{B}_{22}-\mathcal{B}_{33} & \mathcal{B}_{12} & \mathcal{B}_{13} \\
\mathcal{B}_{21} & \mathcal{B}_{22} & \mathcal{B}_{23} \\
\mathcal{B}_{31} & \mathcal{B}_{32} & \mathcal{B}_{33}
\end{array}\right), \\
& \mathcal{B}_{12}=-\frac{2\left(v_{1}^{2}+1\right)\left(v_{0}^{2}-1\right)^{2}\left(v_{-1}^{2}+1\right)}{Z_{0} Z_{1}}\left(\frac{\lambda_{1} \lambda_{2}^{2}}{\lambda-\lambda_{1} \lambda_{2}^{2}}+1\right), \\
& \mathcal{B}_{13}=-\frac{2 \lambda_{1} \lambda_{2}\left(v_{0}^{2}-1\right)\left(v_{-1}^{2}+1\right)}{\left(\lambda+\lambda_{1} \lambda_{2}^{2}\right) Z_{0}}+\frac{\left.4 \lambda_{1} \lambda_{2} v_{-1}\left(v_{0}^{2}-1\right)^{2}\right)\left(v_{1}^{2}+1\right)}{\left(\lambda-\lambda_{1} \lambda_{2}^{2}\right) Z_{0} Z_{1}}, \\
& \mathcal{B}_{21}=-\frac{8\left(v_{0}^{2}+1\right)\left(v_{-2}^{2}+1\right) v_{-1}^{2}}{Z_{0} Z_{-1}}\left(\frac{\lambda_{1} \lambda_{2}^{2}}{\lambda+\lambda_{1} \lambda_{2}^{2}}-1\right) \\
& \mathcal{B}_{22}=-\frac{2 \lambda_{1} \lambda_{2}^{2}}{3}\left(\frac{1}{\lambda+\lambda_{1} \lambda_{2}^{2}}+\frac{1}{\lambda-\lambda_{1} \lambda_{2}^{2}}\right)+\frac{2 \lambda_{1} \lambda_{2}^{2}\left(v_{0}^{2}+1\right)\left(v_{-1}^{2}+1\right)}{\left(\lambda-\lambda_{1} \lambda_{2}^{2}\right) Z_{0}} \\
& +\frac{\left(v_{0}-1\right)^{2} v_{-1}^{2}+5 v_{0}^{2}-2 v_{0}+5}{Z_{0}}+\frac{4 v_{0}\left(v_{0}-1\right)^{2}\left(v_{-1}^{2}+1\right)}{Z_{0} Z_{1}} \\
& -\frac{4\left(2 v_{-1}^{2} v_{-2}+\left(v_{-2}-1\right)^{2}\right)\left(v_{0}^{2}+1\right)}{Z_{0} Z_{-1}}, \\
& \mathcal{B}_{23}=\frac{8 \lambda_{1} \lambda_{2} v_{-1}^{2}\left(v_{0}^{2}-1\right)\left(v_{-2}^{2}+1\right)}{\left(\lambda+\lambda_{1} \lambda_{2}^{2}\right) Z_{-1} Z_{0}}-\frac{4 \lambda_{1} \lambda_{2} v_{-1}\left(v_{0}^{2}+1\right)}{\left(\lambda-\lambda_{1} \lambda_{2}^{2}\right) Z_{n}}, \\
& \mathcal{B}_{31}=-\frac{2 \lambda_{2}\left(v_{0}^{2}+1\right) v_{-1}}{Z_{0}}\left(\frac{2 \lambda_{1} \lambda_{2}^{2}}{\lambda+\lambda_{1} \lambda_{2}^{2}}-1\right)-\frac{4\left(v_{0}^{2}-1\right)\left(v_{-2}^{2}+1\right) v_{-1}}{Z_{-1} Z_{0}}, \\
& \mathcal{B}_{32}=-\frac{2\left(v_{-1}^{2}+1\right)\left(v_{0}^{2}-1\right) \lambda_{1} \lambda_{2}^{2}}{\left(\lambda-\lambda_{1} \lambda_{2}^{2}\right) Z_{0}}+\frac{2 \lambda_{2}\left(2 v_{-1}\left(v_{0}^{2}-v_{0}+1\right)+\left(v_{-1}-1\right)^{2}\right)}{Z_{0}}-\lambda_{2} \\
& +\frac{8 \lambda_{2} v_{-1} v_{0}\left(v_{0}-1\right)^{2}}{Z_{0} Z_{1}}
\end{aligned}
$$




$$
\begin{aligned}
\mathcal{B}_{33} & =-\frac{2}{3} \lambda_{1} \lambda_{2}^{2}\left(1-\frac{6\left(v_{0}^{2}-1\right) v_{-1}}{Z_{n}}\right)\left(\frac{1}{\lambda+\lambda_{1} \lambda_{2}^{2}}+\frac{1}{\lambda-\lambda_{1} \lambda_{2}^{2}}\right), \\
Z_{n} & =\left(v_{n-1}+1\right)^{2} v_{n}^{2}+\left(v_{n-1}-1\right)^{2}, \quad v_{n}=\sqrt{1+e^{2\left(u_{n}-u_{n+1}\right)}}+e^{u_{n}-u_{n+1}} .
\end{aligned}
$$

However, solvability condition 2.18 with the constructed matrices $\mathcal{M}$ and $\mathcal{B}$ is not equivalent to differential-difference equation (1.7). It allows us to find only $\left(u_{1}-u_{0}\right)_{\tau}$. In order to find the matrices giving a compatibility condition equivalent to equation (1.7), we need to employ a gauge transformation, for instance, with the matrix $\mathcal{T}$ :

$$
\mathcal{T}=\left(\begin{array}{ccc}
1 & 0 & 0 \\
0 & e^{u} & 0 \\
0 & 0 & 1
\end{array}\right)
$$

Such transformations correspond to linear changes of the vector function $V$ of form $V=\mathcal{T} U$ and under such change the corresponding matrices $\mathcal{M}$ and $\mathcal{B}$ are transformed by the known formulae.

Theorem 2.3. The Lax pair for the differential-difference equation (1.7) is of form (2.14) with the matrices $\mathcal{T}_{n+1}^{-1} \mathcal{M T}$ and $\mathcal{T}^{-1}\left(\mathcal{B} \mathcal{T}-\mathcal{T}_{\tau}\right)$.

Remark 2.1. Knowing the matrix $\mathcal{B}$, we can also write the operator $B$. However, it is nonlocal because of the presence of the spectral parameter in the denominators of the entries of the matrix $\mathcal{B}$.

2.4. Representation of the operators via shift operator $T$. In the concluding part of our work we write the operators $\hat{M}$ and $\hat{L}$ via the shift operator $T$. In order to do this, we choose the vector function in the form

$$
V=\left(\lambda-\lambda_{1} \lambda_{2}^{2}\right)^{n} e^{u}\left(\begin{array}{c}
-T P \\
P \\
\frac{\lambda-\lambda_{1} \lambda_{2}^{2}}{2 \lambda_{1} \lambda_{2} \sqrt{e^{2 u_{1}-2 u}+1}}\left(-\frac{\lambda+\lambda_{1} \lambda_{2}^{2}}{\lambda-\lambda_{1} \lambda_{2}^{2}} P+\left(e^{2 u_{1}-2 u}+1\right) T P-e^{2 u_{1}-2 u} T^{2} P\right)
\end{array}\right) .
$$

Then in a vector relation $T V=\mathcal{M V}$ two identities hold immediately, while the third relation gives a linear difference equation for the function $P$ :

$$
\left((T-1) \frac{e^{2 u}}{\sqrt{e^{2 u_{1}}+e^{2 u}}}+\sqrt{e^{2 u_{1}}+e^{2 u}} T\right) \Lambda P+\left((T-1) \frac{e^{2 u_{1}}}{\sqrt{e^{2 u_{1}}+e^{2 u}}}-\sqrt{e^{2 u_{2}}+e^{2 u_{1}}}\right) T^{2} P=0,
$$

where $\Lambda=\frac{\lambda+\lambda_{1} \lambda_{2}^{2}}{\lambda-\lambda_{1} \lambda_{2}^{2}}$ is new spectral parameter. Expressing then formally $\Lambda P$, we obtain the operator $\hat{M}$ in a non-local form:

$$
\hat{M}=\left((T-1) \frac{e^{2 u}}{\sqrt{e^{2 u_{1}}+e^{2 u}}}+\sqrt{e^{2 u_{1}}+e^{2 u}} T\right)^{-1}\left((T-1) \frac{e^{2 u_{1}}}{\sqrt{e^{2 u_{1}}+e^{2 u}}}-\sqrt{e^{2 u_{2}}+e^{2 u_{1}}}\right) T^{2} .
$$

The operator $\hat{L}$ is found by the relation $\frac{d V}{d x}=\mathcal{L} V$ and its second component is of the form

$$
\frac{d P}{d x}=\left(\lambda_{1} e^{-2 u}-u_{0,1}+\frac{\lambda_{2} e^{2 u}}{\sqrt{e^{2 u_{1}}+e^{u}}}\right) P-\frac{\lambda_{2}}{\Lambda-1} \frac{e^{2 u} P-\left(e^{2 u_{1}}+e^{2 u}\right) T P+e^{2 u_{1}} T^{2} P}{\sqrt{e^{2 u_{1}}+e^{u}}} .
$$

The operator $\hat{L}$ defines a differentiation operator and it is of the form

$$
\hat{L}=\left(\lambda_{1} e^{-2 u}-u_{0,1}+\frac{\lambda_{2} e^{2 u}}{\sqrt{e^{2 u_{1}}+e^{u}}}\right)-\lambda_{2} \frac{(T-1) e^{2 u}(T-1)}{\sqrt{e^{2 u_{1}}+e^{u}}}(\hat{M}-1)^{-1} .
$$

The compatibility condition of the operators $\hat{M}$ and $\hat{L}$ reads as:

$$
\frac{d \hat{M}}{d x}+[\hat{M}, \hat{L}]=0 .
$$


It is equivalent to equation (1.1). These operators are useful for finding the conservation laws, see, for instance, [11].

\section{BIBLIOGRAPHY}

1. R.N.Garifullin, I.T.Habibullin. Generalized symmetries and integrability conditions for hyperbolic type semi-discrete equations // J. Phys. A: Math. Theor. 54:20, 205201 (2021).

2. G. Tzitzeica. Sur une nouvelle classe de surfaces // Rendiconti del Circolo Matematico di Palermo 25:1, 180-187 (1907).

3. A.V. Zhiber, A.B. Shabat. Klein-Gordon equations with a nontrivial group // Dokl. AN SSSR. 247:5, 1103-1107 (1979). [Soviet Phys. Dokl. 24, 607-609 (1979).]

4. A.P. Veselov, A.B. Shabat. Dressing chains and spectral theory of the Schrödinger operator // Funkts. Anal. Pril. 27:2, 1-21 (1993). [Funct. Anal. Appl. 27:2, 81-96 (1993).]

5. R. Yamilov. Symmetries as integrability criteria for differential difference equations // J. Phys. A: Math. Gen. 39(45), R541 (2006).

6. R.I. Yamilov. Classification of discrete evolution equations // Uspekhi Matem. Nauk 38:6, 155156 (1983). (in Russian).

7. R.I. Yamilov. Invertible changes of variables generated by Backlund transformations // Teor. Matem. Fiz. 85:3, 368-375 (1990). [Theor. Math. Phys. 85:3, 1269-1275 (1990).]

8. A.G. Meshkov, V.V. Sokolov. Integrable evolution equations with a constant separant // Ufimskij Matem. Zhurn. 4:3, 104-154 (2012). [Ufa Math. J. 4:3, 104-152 (2012).]

9. D.J. Kaup. On the inverse scattering problem for cubic eigenvalue problems of the class $\psi_{x x x}+6 Q \psi_{x}+6 R \psi=\lambda \psi / /$ Stud. Appl. Math. 62:3, 189-216 (1980).

10. A.P. Fordy, J. Gibbons. Factorization of operators I. Miura transformations // J. Math. Phys. 21:10, 2508-2510 (1980).

11. R.N. Garifullin, R.I. Yamilov. On integrability of a discrete analogue of Kaup-Kupershmidt equation // Ufimskij Matem. Zhurn. 9:3, (2017). [Ufa Math. J. 9:3, 158-164 (2017).]

Rustem Nailevich Garifullin,

Institute of Mathematics,

Ufa Federal Research Center, RAS

Chernyshevsky str. 112,

450008, Ufa, Russia

E-mail: rustem@matem.anrb.ru 